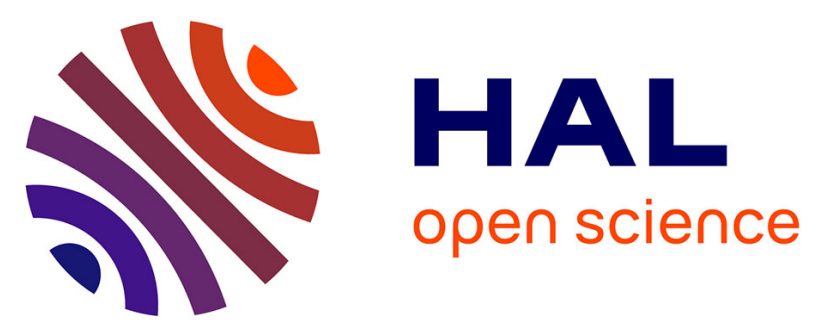

\title{
A multi-scale approach for the optimum design of sandwich plates with honeycomb core. Part II: the optimisation strategy
}

\author{
Anita Catapano, Marco Montemurro
}

\section{- To cite this version:}

Anita Catapano, Marco Montemurro. A multi-scale approach for the optimum design of sandwich plates with honeycomb core. Part II: the optimisation strategy. Composite Structures, 2014, 118, pp.677-690. 10.1016/j.compstruct.2014.07.058 . hal-01063071

\section{HAL Id: hal-01063071 \\ https://hal.science/hal-01063071}

Submitted on 5 Sep 2017

HAL is a multi-disciplinary open access archive for the deposit and dissemination of scientific research documents, whether they are published or not. The documents may come from teaching and research institutions in France or abroad, or from public or private research centers.
L'archive ouverte pluridisciplinaire HAL, est destinée au dépôt et à la diffusion de documents scientifiques de niveau recherche, publiés ou non, émanant des établissements d'enseignement et de recherche français ou étrangers, des laboratoires publics ou privés. 


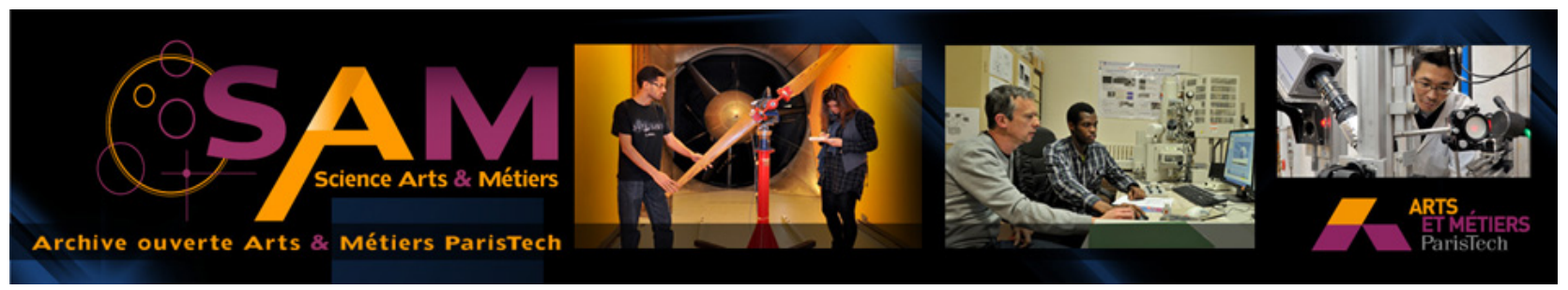

Science Arts \& Métiers (SAM)

is an open access repository that collects the work of Arts et Métiers ParisTech researchers and makes it freely available over the web where possible.

This is an author-deposited version published in: http://sam.ensam.eu

Handle ID: .http://hdl.handle.net/10985/8493

\section{To cite this version :}

Anita CATAPANO, Marco MONTEMURRO - A multi-scale approach for the optimum design of sandwich plates with honeycomb core. Part II: the optimisation strategy - Composite Structures p.14p. -2014 


\title{
A multi-scale approach for the optimum design of sandwich plates with honeycomb core. Part II: the optimisation strategy
}

\author{
Anita Catapano ${ }^{\mathrm{a}}$, Marco Montemurro ${ }^{\mathrm{b}, *}$ \\ a Université de Bordeaux, I2M CNRS UMR 5295, F-33400 Talence, France \\ ${ }^{\mathrm{b}}$ Arts et Métiers ParisTech, I2M CNRS UMR 5295, F-33400 Talence, France
}

Keywords:

Sandwich structures

Optimisation

Genetic algorithms

Buckling

Structural design

Composite materials

\begin{abstract}
A B S T R A C T
This work deals with the problem of the optimum design of a sandwich panel. The design strategy that we propose is a numerical optimisation procedure that does not make any simplifying assumption to obtain a true global optimum configuration of the system. To face the design of the sandwich structure at both meso and macro scales, we use a two-level optimisation strategy: at the first level we determine the optimal geometry of the unit cell of the core together with the material and geometric parameters of the laminated skins, while at the second level we determine the optimal skins lay-up giving the geometrical and material parameters issued from the first level. The two-level strategy relies both on the use of the polar formalism for the description of the anisotropic behaviour of the laminates and on the use of a genetic algorithm as optimisation tool to perform the solution search. To prove its effectiveness, we apply our strategy to the least-weight design of a sandwich plate, satisfying several constraints: on the first buckling load, on the positive-definiteness of the stiffness tensor of the core, on the ratio between skins and core thickness and on the admissible moduli for the laminated skins.
\end{abstract}

\section{Introduction}

Sandwich panels are increasingly used in aerospace, automotive and naval industries thanks to their high stiffness-to-weight and strength-to-weight ratios. In order to have a further weight reduction when employing this kind of structures, in aerospace applications sandwich panels are composed by glass or carbonfiber composite skins separated by aluminium or resin honeycombs, or by polymer foams. In addition, material and geometrical properties can be designed to provide sandwich plates with different stiffness and density characteristics.

The optimum design of sandwich structures is much more cumbersome than that of a classical monolithic structure. The difficulties increase when the sandwich structure is made of composite skins and a honeycomb core. In this case we have to face, into the same design process, both the difficulty of designing a laminated plate (concerning the skins) and the difficulty of designing a complex 3D cellular continuum such as the honeycomb core. Therefore, engineers always use some simplifying assumptions or rules to obtain, in an easier and faster way, a solution. For example, in [1-3] the optimal design of a sandwich plate is addressed determining exclusively the optimum thickness of both the core and the skins, keeping constant the rest of geometric and material parameters of the system.

Triantafillou and Gibson [4] gave the analytical relations to determine the skins and core thickness and the core density which minimise the weight of a foam core sandwich beam for a given strength. In $[5,6]$ the minimum weight design of sandwich panels under uniaxial compressive loading conditions is solved analytically using the principle of the most efficient chain wherein each link fails simultaneously. The optimisation parameters were the geometry of the unit cell and the thickness of the skins. In this way, also the density of the core is introduced among the design variables. Another analytical study for the minimum weight design of foam-core sandwich panels under stiffness and strength requirements is presented in [7]. Here the three design variables were the thickness of the core and that of the skins (assumed to be identical) along with the core density. A semi-analytical method to minimise the density of truss core structures under prescribed constraints on strength and stiffness is addressed in [8]. However, as it is classical in purely analytical-based approaches, the relations giving rise to the optimal values of the geometric variables are obtained thanks to the imposition of particular load cases (for example uniaxial) and/or boundary conditions (BCs) such as simply supported or 


\section{Notations}

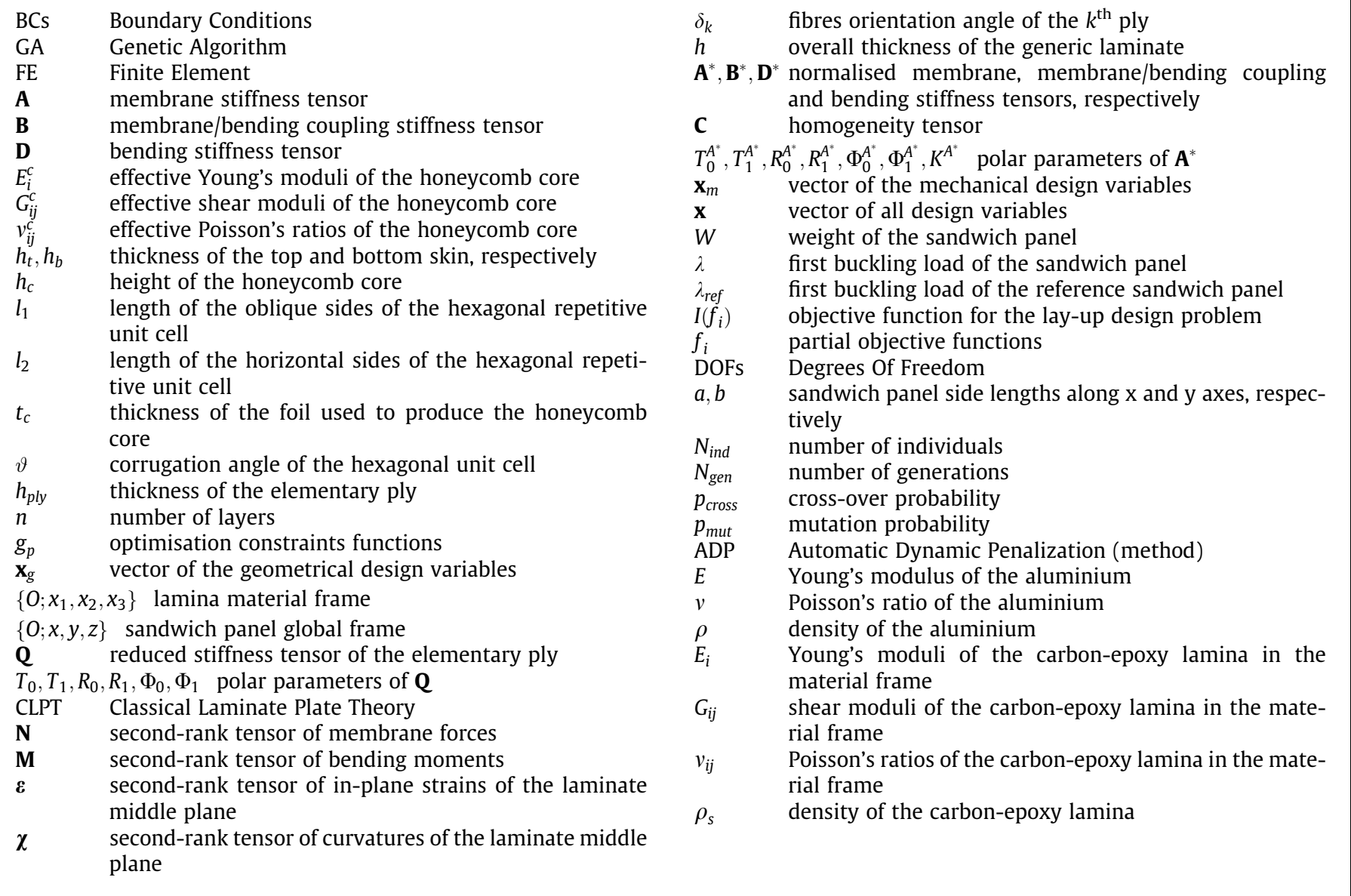

clamped plate. On the other hand, in the case of more complex BCs it is not possible to obtain an analytical solution, therefore a numerical strategy is needed.

A step further in the optimum design of sandwich panels with corrugated cores was done in [9]. The authors dealt with the problem of the least-weight design of a sandwich plate considering as design variables the thickness of the cell walls as well as that of the skins together with the total height of the panel. They used an analytical model to evaluate both the buckling load of the core and the faces yielding which were considered as optimisation constraints. The optimisation problem was solved using a Genetic Algorithm (GA). Wennhange conducted an interesting work on the weight minimisation of sandwich structures under acoustic constraints. He firstly developed a semi-analytical model in [10], a subsequent experimental verification in [11] and an application to a real-world engineering problem concerning the design of a railway car body in [12]. Other studies on numerical strategies for the optimal design of sandwich structures can be found in [13] for the maximisation of blast load mitigation, in [14] for the optimisation of the head impact mitigation, in [15] where the transverse shear stiffness of the panel is maximised and in [16] for the minimisation of both mass and costs of composite sandwich structures for rail vehicle floor panels.

The objective of the present work is twofold: on one hand, we want to formulate and solve the problem of designing a sandwich panel as an optimisation problem on different scales and, on the other hand, we want to include within the same design process the full set of geometrical and material parameters defining the behaviour of the structure (at each scale) as optimisation variables. In this regard, we propose a very general design strategy that consists in a numerical optimisation procedure without simplifying hypothesis to obtain a true optimal configuration of the system. The design process that we propose is not submitted to restrictions, indeed any parameter characterising our structure is an optimisation variable: the geometry of the unit cell of the core along with the number as well as the orientation angle of the plies for each skin.

In order to deal with the design problem of the sandwich plate at both meso and macro scales, we used a two-level optimisation strategy. At the first level we determine the optimum geometry of the unit cell (core meso-scale) together with the material and geometric parameters of the laminated skins (at this level the laminate representing each skin is modelled as an equivalent homogeneous anisotropic plate whose behaviour at the macro-scale is described in terms of laminate polar parameters, see [17]). At the second level of the strategy, we determine the optimal skins layup (the skin meso-scale) meeting the optimal combination of their material and geometrical parameters resulting from the first level of the strategy. The whole procedure is based on one hand on the use of the polar formalism [18] and on the other hand on the new version of the GA BIANCA [19-21].

Since the first level of the strategy involves two different scales (the macro-scale of the sandwich panel as well as the meso-scale of the honeycomb core) we conceived an appropriate model of the repetitive unit cell of the core able to properly evaluate its effective elastic properties used at the macro-scale. This model has been discussed in Part I of the present work. In the first paper we presented the numerical homogenisation technique as well as the related 3D finite element model of the unit cell used within the first level of the optimisation strategy to determine the 
effective material properties of the honeycomb core which is modelled, at the macro-scale, as an equivalent homogeneous orthotropic continuum. In this second paper we will focus on the description of the two-level optimisation strategy along with some numerical examples in order to prove its effectiveness.

The paper is organised as follows: the design problem as well as the two-level strategy are discussed in Section 2. The mathematical formulation of the first-level problem is detailed in Section 3, while the problem of determining a suitable laminate is formulated in Section 4. A concise description of the Finite Element (FE) models of the sandwich structure at both meso and macro scales are given in Section 5 while in Section 6 we show the numerical results of the optimisation procedure. Finally, Section 7 ends the paper with some concluding remarks.

\section{Optimal design of sandwich panels with honeycomb core}

\subsection{Description of the problem}

The optimisation strategy presented in this work is applied to a sandwich plate composed by two laminated skins and a metal honeycomb core with hexagonal cells as depicted in Fig. 1. The skins are made of carbon-epoxy unidirectional orthotropic laminae while the honeycomb core is obtained from aluminium alloy foils, see Table 1 for the material properties derived from $[22,23]$.

Concerning the honeycomb core, the basic classical assumptions used to evaluate its elastic response and, hence, to determine its effective material properties (at the macro-scale) are:

- linear, elastic behaviour for the material of the cell walls;

- perfect bonding for the wall-to-wall contact;

- the buckling of the cell walls is disregarded.

Concerning the mechanical behaviour (at the macro-scale) of the two laminated skins they are modelled, for obvious mechanical reasons, as quasi-homogeneous fully orthotropic laminates, see Section 3.2 .

In addition, no simplifying hypotheses are made on the geometric and mechanical parameters of both skins and core. Only avoiding the use of a priori assumptions that extremely shrink the solution space (e.g. the use of symmetric balanced stacks for the skins laminates to attain membrane/bending uncoupling and membrane orthotropy, respectively, or the use of regular hexagonal cells to reduce the number of optimisation variables for the core and to deal, at the macro-scale, with a transverse isotropic cellular solid) one can hope to obtain the true global optimum for a given problem: this is a key-point in our approach.

\subsection{Description of the multi-scale two-level optimisation strategy}

The goal of our design strategy is the minimisation of the weight of the sandwich plate subject to constraints of different

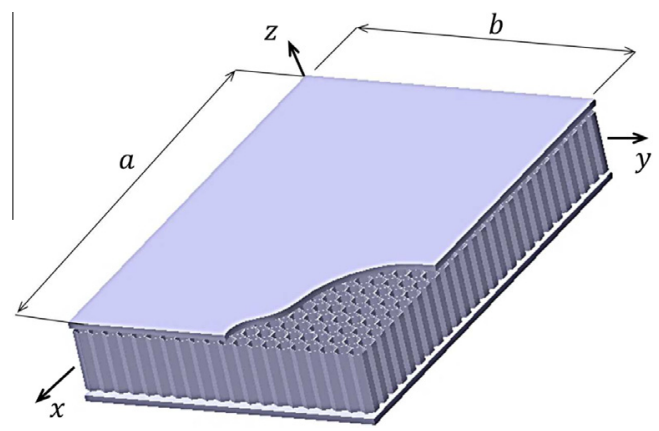

Fig. 1. Geometry of the sandwich panel.
Table 1

Material properties of the aluminium foil of the core and of the carbon-epoxy laminae of the skins.

\begin{tabular}{|c|c|c|c|}
\hline \multicolumn{2}{|c|}{ Aluminium } & \multicolumn{2}{|c|}{ Carbon-Epoxy } \\
\hline \multicolumn{4}{|c|}{ Material properties } \\
\hline$E$ & $70000 \mathrm{MPa}$ & $E_{1}$ & $181000 \mathrm{MPa}$ \\
\hline$v$ & 0.33 & $E_{2}$ & $10300 \mathrm{MPa}$ \\
\hline \multirow{4}{*}{\multicolumn{2}{|c|}{$2.7 \times 10^{-6} \mathrm{~kg} / \mathrm{mm}^{3}$}} & $G_{12}$ & $7170 \mathrm{MPa}$ \\
\hline & & $v_{12}$ & 0.28 \\
\hline & & $\rho_{s}$ & $1.58 \times 10^{-6} \mathrm{~kg} / \mathrm{mm}^{3}$ \\
\hline & & $h_{p l y}$ & $0.125 \mathrm{~mm}$ \\
\hline \multicolumn{4}{|c|}{ Polar parameters } \\
\hline & & $T_{0}$ & $26880 \mathrm{MPa}$ \\
\hline & & $T_{1}$ & $24744 \mathrm{MPa}$ \\
\hline & & $R_{0}$ & $19710 \mathrm{MPa}$ \\
\hline & & $R_{1}$ & $21433 \mathrm{MPa}$ \\
\hline & & $\Phi_{0}, \Phi_{1}$ & $0 \mathrm{deg}$ \\
\hline
\end{tabular}

nature, i.e. mechanical, geometrical as well as feasibility constraints. The proposed optimisation procedure is articulated into two distinct (but linked) problems as described here below.

\subsubsection{First-level problem}

The aim of this phase is the determination of the optimal geometry of the unit cell together with the material and geometric parameters of the laminated skins in order to minimise the weight of the structure and to satisfy, simultaneously, the full set of optimisation constraints. At this level the laminate representing each skin is modelled as an equivalent homogeneous anisotropic plate whose behaviour at the macro-scale is described in terms of laminate polar parameters, see [17,20,24], by means of the classical stiffness tensors A, B and D (membrane, membrane/bending coupling and bending stiffness, respectively). Concerning the model of the honeycomb core, the first-level problem involves two different scales: the meso-scale of the repetitive unit cell characterised by its geometric variables, as well as the macro-scale where the core itself is modelled as an homogeneous orthotropic solid. Therefore, the link between these two scales is represented by the homogenisation phase of the honeycomb core (see Part I) that allows us to represent it, at the macro-scale level, as a homogeneous orthotropic continuum characterised by its equivalent material properties (which depend upon the geometric parameters of the unit cell).

\subsubsection{Second-level problem}

At the second level of the strategy, we have to determine the optimal lay-up for both skins (the skin meso-scale) meeting the optimal combination of their material and geometrical parameters provided by the first level of the strategy. The goal of this phase is, hence, to find at least one stacking sequence, for each skin, which has to be quasi-homogeneous, fully orthotropic and that has to satisfy the optimal polar parameters resulting from the first step. At this level of the strategy, the design variables are the layer orientations.

\section{Mathematical formulation of the first-level problem}

The overall characteristics of the optimal structure have to be designed during this phase. The weight minimisation of the sandwich plate will be done satisfying, on one side, the constraint on the first buckling load and, on the other side, the geometric constraints on the ratio between skins and core thickness along with some mechanical constraints on the elastic moduli of both core and skins. These aspects are described in detail in the following subsections.

\subsection{Geometrical design variables}

Before specifying the mathematical formulation of the first-level problem, we introduce the design variables which are 


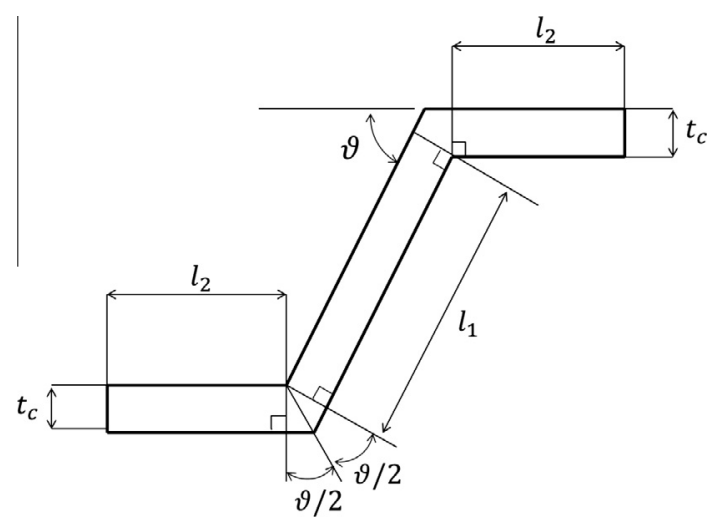

Fig. 2. Geometrical parameters of the unit cell of the honeycomb core

of two types: geometrical and mechanical. Concerning the geometrical design variables, they are:

- the thickness of both top and bottom skins, $h_{t}$ and $h_{b}$ respectively;

- the thickness of the core $h_{c}$;

- the geometrical parameters of the unit cell of the honeycomb core $l_{1}, l_{2}, t_{c}$ and $\vartheta$, see Fig. 2 .

The geometrical and material design variables along with their nature and bounds for the first-level problem are detailed in Table 2. At this level of the optimisation procedure, the thickness of the laminated skins is considered as a discrete optimisation variable, the discretisation step being equal to the thickness of the elementary layer used for the fabrication of the laminate, i.e. $\Delta h_{t}=\Delta h_{b}=h_{p l y}$ (see Table 1 ). This assumption responds to a technological constraint, and, in addition, the optimal value of these parameters will give us also the optimal number of layers $n$ to be used during the second-level design procedure.

Moreover, in order to obtain a "true" sandwich panel, i.e. a plate characterised by a thick core and thin skins, we introduced a geometrical constraint on the ratio between the thickness of each skin and that of the core. Such constraints can be written as follows:

$g_{1}\left(\mathbf{x}_{\mathbf{g}}\right)=\frac{h_{t}}{h_{c}}-\frac{1}{20} \leqslant 0$,

$g_{2}\left(\mathbf{x}_{\mathbf{g}}\right)=\frac{h_{b}}{h_{c}}-\frac{1}{20} \leqslant 0$

where $\mathbf{x}_{\mathbf{g}}=\left\{\vartheta, t_{c}, l_{2}, l_{1}, h_{c}, h_{t}, h_{b}\right\}$ is the vector of the geometrical design variables of the problem. Together with the previous ones, we have to add further constraints to ensure the positive definiteness of the stiffness matrix of the honeycomb core whose effective elastic properties depend on the geometric parameters of the unit cell. These constraints can be written as follows (see [25] for more details):

$g_{3}\left(\mathbf{x}_{\mathbf{g}}\right)=-E_{1}^{c}<0$,

$g_{4}\left(\mathbf{x}_{\mathbf{g}}\right)=-E_{2}^{c}<0$

$g_{5}\left(\mathbf{x}_{\mathbf{g}}\right)=-E_{3}^{c}<0$

$g_{6}\left(\mathbf{x}_{\mathbf{g}}\right)=-G_{12}^{c}<0$

$g_{7}\left(\mathbf{x}_{\mathbf{g}}\right)=-G_{13}^{c}<0$

$g_{8}\left(\mathbf{x}_{\mathbf{g}}\right)=-G_{23}^{c}<0$,

$g_{9}\left(\mathbf{x}_{\mathbf{g}}\right)=\left|v_{12}^{c}\right|-\sqrt{\frac{E_{1}^{c}}{E_{2}^{c}}}<0$,

$g_{10}\left(\mathbf{x}_{\mathbf{g}}\right)=\left|v_{13}^{c}\right|-\sqrt{\frac{E_{1}^{c}}{E_{3}^{c}}}<0$,

$g_{11}\left(\mathbf{x}_{\mathbf{g}}\right)=\left|v_{23}^{c}\right|-\sqrt{\frac{E_{2}^{c}}{E_{3}^{c}}}<0$,

$g_{12}\left(\mathbf{x}_{\mathbf{g}}\right)=2 v_{12}^{c} v_{13}^{c} v_{23}^{c} \frac{E_{3}^{c}}{E_{1}^{c}}+\left(v_{12}^{c}\right)^{2} \frac{E_{2}^{c}}{E_{1}^{c}}+\left(v_{23}^{c}\right)^{2} \frac{E_{3}^{c}}{E_{2}^{c}}+\left(v_{13}^{c}\right)^{2} \frac{E_{3}^{c}}{E_{1}^{c}}<0$.
The terms $E_{1}^{c}, E_{2}^{c}, E_{3}^{c}, G_{12}^{c}, G_{13}^{c}, G_{23}^{c}, v_{12}^{c}, v_{13}^{c}$ and $v_{23}^{c}$ are the effective material properties (engineering moduli) of the homogeneous orthotropic honeycomb core and they are determined via the numerical homogenisation phase detailed in Part I of the present work. Therefore, Eq. (2) represent a set of optimisation constraints indirectly applied on the geometrical variables of the repetitive unit of the honeycomb core.

\subsection{Mechanical design variables}

Concerning the mechanical variables we use the polar formalism which gives a representation of any planar tensor by means of a complete set of independent invariants, i.e. the polar parameters. Using the polar formalism, the representation of the reduced stiffness tensor $\mathbf{Q}$ for an anisotropic layer expressed in the lamina material frame $\left\{0 ; x_{1}, x_{2}, x_{3}\right\}$ (using Voigt's notation) is:

$$
\left\{\begin{array}{l}
Q_{11}=T_{0}+2 T_{1}+R_{0} \cos 4 \Phi_{0}+4 R_{1} \cos 2 \Phi_{1}, \\
Q_{16}=R_{0} \sin 4 \Phi_{0}+2 R_{1} \sin 2 \Phi_{1}, \\
Q_{12}=-T_{0}+2 T_{1}-R_{0} \cos 4 \Phi_{0}, \\
Q_{66}=T_{0}-R_{0} \cos 4 \Phi_{0}, \\
Q_{26}=-R_{0} \sin 4 \Phi_{0}+2 R_{1} \sin 2 \Phi_{1}, \\
Q_{22}=T_{0}+2 T_{1}+R_{0} \cos 4 \Phi_{0}-4 R_{1} \cos 2 \Phi_{1},
\end{array}\right.
$$

where $T_{0}, T_{1}, R_{0}, R_{1}$ and $\left(\Phi_{0}-\Phi_{1}\right)$ are the polar tensor invariants. $T_{0}$ and $T_{1}$ are linked to the isotropic part of the tensor (the isotropic moduli), $R_{0}$ and $R_{1}$ are linked to the anisotropic part of the tensor (the anisotropic moduli), whilst $\Phi_{0}$ and $\Phi_{1}$ are the polar angles (that give the direction of the main axes of the tensor). The advantages of the polar formalism are at least three: firstly, the tensor is expressed (by means of a complex-variable transformation) through a set of tensor invariants, secondly each invariant is linked to a precise elastic symmetry (for example the tensor is isotropic when $R_{0}=R_{1}=0$ or it shows the so-called square symmetry when $R_{1}=0$, etc.) and, finally, any rotation of the tensor can be easily expressed by subtracting the rotation angle from the polar angles $\Phi_{0}$ and $\Phi_{1}$. For more details on the polar formalism the reader is addressed to $[17,18]$.

The constitutive law of a laminate in the framework of the Classical Laminate Plate Theory (CLPT) is:

$$
\begin{aligned}
& \mathbf{N}=\mathbf{A} \boldsymbol{\varepsilon}+\mathbf{B} \boldsymbol{\chi}, \\
& \mathbf{M}=\mathbf{B} \boldsymbol{\varepsilon}+\mathbf{D} \boldsymbol{\chi} .
\end{aligned}
$$

where $\mathbf{N}$ and $\mathbf{M}$ are the second-rank tensors representing the membrane forces and the bending moments, respectively, $\boldsymbol{\varepsilon}$ and $\chi$ are the second-rank tensors of in-plane strains and curvatures of the laminate middle plane, whilst A,D and B are the fourth-rank tensors of membrane, bending and membrane/bending coupling stiffness, respectively. The composition laws of tensors $\mathbf{A}, \mathbf{B}$ and $\mathbf{D}$ for a laminate composed by $n$ plies are:

$$
\begin{aligned}
& \mathbf{A}=\sum_{k=1}^{n} \mathbf{Q}_{k}\left(\delta_{k}\right)\left(z_{k}-z_{k-1}\right), \\
& \mathbf{B}=\frac{1}{2} \sum_{k=1}^{n} \mathbf{Q}_{k}\left(\delta_{k}\right)\left(z_{k}^{2}-z_{k-1}^{2}\right), \\
& \mathbf{D}=\frac{1}{3} \sum_{k=1}^{n} \mathbf{Q}_{k}\left(\delta_{k}\right)\left(z_{k}^{3}-z_{k-1}^{3}\right) .
\end{aligned}
$$

$\delta_{k}$ represents the fibres orientation angle of the $k^{\text {th }}$ ply with respect to the laminate global frame, whilst $z_{k}$ and $z_{k-1}$ represent the $z$ coordinate of the top and bottom surface of the $k^{\text {th }}$ ply, respectively, see [25]. It is useful to introduce the normalised stiffness tensors as follows:

$$
\mathbf{A}^{*}=\frac{\mathbf{A}}{h}, \quad \mathbf{B}^{*}=\frac{2 \mathbf{B}}{h^{2}}, \quad \mathbf{D}^{*}=\frac{12 \mathbf{D}}{h^{3}}
$$


Table 2

Design space of the first-level problem.

\begin{tabular}{|c|c|c|c|c|}
\hline Design variable & Type & Lower bound & Upper bound & Discretisation step \\
\hline$\vartheta$ [deg] & Discrete & 0 & 90 & 1 \\
\hline$t_{c}[\mathrm{~mm}]$ & Discrete & 0.03 & 0.07 & 0.001 \\
\hline$l_{2}[\mathrm{~mm}]$ & Discrete & 0.3 & 3.5 & 0.01 \\
\hline$l_{1}[\mathrm{~mm}]$ & Discrete & 0.6 & 14 & 0.1 \\
\hline$h_{c}[\mathrm{~mm}]$ & Discrete & 25 & 150 & 1 \\
\hline$\left(R_{0 K}^{A^{*}}\right)_{t}[\mathrm{MPa}]$ & Continuous & -19710.0 & 19710.0 & - \\
\hline$\left(R_{1}^{A^{*}}\right)_{t}[\mathrm{MPa}]$ & Continuous & 0 & 21433.0 & - \\
\hline$\left(\Phi_{1}^{A^{*}}\right)_{t}[\operatorname{deg}]$ & Discrete & -90 & 90 & 1 \\
\hline$h_{t}[\mathrm{~mm}]$ & Discrete & 3 & 6 & 0.125 \\
\hline$\left(R_{0 K}^{A^{+}}\right)_{b}[\mathrm{MPa}]$ & Continuous & -19710.0 & 19710.0 & - \\
\hline$\left(R_{1}^{A^{*}}\right)_{b}[\mathrm{MPa}]$ & Continuous & 0 & 21433.0 & - \\
\hline$\left(\Phi_{1}^{A^{*}}\right)_{b}[\operatorname{deg}]$ & Discrete & -90 & 90 & 1 \\
\hline$h_{b}[\mathrm{~mm}]$ & Discrete & 3 & 6 & 0.125 \\
\hline
\end{tabular}

where $h$ is the overall thickness of the laminate. The conditions for quasi-homogeneity and orthotropy are expressed as, see [17,19]:

$$
\begin{aligned}
& \mathbf{B}^{*}=\mathbf{0}, \\
& \mathbf{C}=\mathbf{A}^{*}-\mathbf{D}^{*}=\mathbf{0}, \\
& \Phi_{0}^{A^{*}}-\Phi_{1}^{A^{*}}=K^{A^{*}} \frac{\pi}{4},
\end{aligned}
$$

where $\mathbf{C}$ is the homogeneity tensor whilst $K^{A^{*}}$ is the parameter giving the shape of the orthotropy, that can get the values 0 or 1 , see [17]. The quasi-homogeneity condition ensures that the laminate has identical in-plane and bending behaviours: in this case the design domain of the elastic moduli is the same for in-plane and bending normalised stiffness tensors, and one single set of polar invariants can describe both elastic behaviours. Quasi-homogeneous laminates are a well-known class of laminates, that are widely used in the design of membrane as well as flexural properties, see [26]. All the previous tensors can be expressed through the polar formalism. For instance, the expression of the polar parameters of tensor $\mathbf{A}^{*}$ in terms of those of the constitutive layers, for an orthotropic laminate composed by identical plies, is:

$T_{0}^{A^{*}}=T_{0}$,

$T_{1}^{A^{*}}=T_{1}$,

$(-1)^{K^{A^{*}}} R_{0}^{A^{*}} e^{4 i \Phi_{1}^{A^{*}}}=\frac{1}{n}(-1)^{K} R_{0} \sum_{j=1}^{n} e^{4 i \delta_{j}}$,

$R_{1}^{A^{*}} e^{2 i \Phi_{1}^{A^{*}}}=\frac{1}{n} R_{1} \sum_{j=1}^{n} e^{2 i \delta_{j}}$.

Eq. (8) shows that in the case of a laminate composed by identical plies the isotropic moduli of the in-plane normalised stiffness tensor $\mathbf{A}^{*}$ are equal to those of the elementary layer, thus they do not take part into the optimisation process as design variables, see $[17,19,27]$. Moreover we can introduce the quantity

$R_{0 K}^{A^{*}}=(-1)^{K^{A^{*}}} R_{0}^{A^{*}}$,

which is obtained combining the two invariants $R_{0}^{A^{*}}$ and $K^{A^{*}}$. Therefore, taking into account all of the previous considerations and thanks to quasi-homogeneity and orthotropy conditions, see Eq. (7), we can reduce to only three the total number of mechanical design variables describing the behaviour of each laminated skin: the anisotropic polar parameters $R_{0 K}^{A^{*}}$ and $R_{1}^{A^{*}}$ and the polar angle $\Phi_{1}^{A^{*}}$ that represents the orientation of the main orthotropy axis.

In addition, in the formulation of the optimisation problem for the first level of the strategy, we have also to impose the geometric and feasibility constraints on the polar parameters, which arise from the combination of the layers orientations and positions within the stack. These constraints ensure that the obtained optimal polar parameters correspond to a feasible laminate that will be designed during the second step of the optimisation strategy, see [28]. Since the laminate is quasi-homogeneous, such constraints can be written only for tensor $\mathbf{A}^{*}$ as follows:

$\left\{\begin{array}{l}-R_{0} \leqslant R_{0 K}^{A^{*}} \leqslant R_{0}, \\ 0 \leqslant R_{1}^{A^{*}} \leqslant R_{1}, \\ 2\left(\frac{R_{1}^{A^{*}}}{R_{1}}\right)^{2}-1-\frac{R_{0 K}^{A^{*}}}{R_{0}} \leqslant 0 .\end{array}\right.$

Of course, the previous equations must be written for the mechanical design variables of both top and bottom skins which are: the three polar parameters of the top skin, namely $\left(R_{0 K}^{A^{*}}\right)_{t},\left(R_{1}^{A^{*}}\right)_{t}$ and $\left(\Phi_{1}^{A^{*}}\right)_{t}$ and the corresponding ones of the bottom skin, i.e. $\left(R_{0 K}^{A^{*}}\right)_{b},\left(R_{1}^{A^{*}}\right)_{b}$ and $\left(\Phi_{1}^{A^{*}}\right)_{b}$. The previous variables can be grouped into the vector of mechanical design variables as follows: $\mathbf{X}_{\mathbf{m}}=\left\{\left(R_{0 K}^{A^{*}}\right)_{t},\left(R_{1}^{A^{*}}\right)_{t},\left(\Phi_{1}^{A^{*}}\right)_{t},\left(R_{0 K}^{A^{*}}\right)_{b},\left(R_{1}^{A^{*}}\right)_{b},\left(\Phi_{1}^{A^{*}}\right)_{b}\right\}$.

First and second constraints of Eq. (10) can be taken into account as admissible intervals for the relevant optimisation variables, i.e. on $\left(R_{0 K}^{A^{*}}\right)_{t},\left(R_{0 K}^{A^{*}}\right)_{b},\left(R_{1}^{A^{*}}\right)_{t}$ and $\left(R_{1}^{A^{*}}\right)_{b}$. Hence, the feasibility constraints imposed to the optimisation problem are:

$g_{13}\left(\mathbf{x}_{\mathbf{m}}\right)=2\left(\frac{\left(R_{1}^{A^{*}}\right)_{t}}{R_{1}}\right)^{2}-1-\frac{\left(R_{0 K}^{A^{*}}\right)_{t}}{R_{0}} \leqslant 0$,

$g_{14}\left(\mathbf{x}_{\mathbf{m}}\right)=2\left(\frac{\left(R_{1}^{A^{*}}\right)_{b}}{R_{1}}\right)^{2}-1-\frac{\left(R_{0 K}^{A^{*}}\right)_{b}}{R_{0}} \leqslant 0$.

For a wide discussion upon the laminate feasibility and geometrical bounds as well as on the importance of the quasi-homogeneity assumption the reader is addressed to [28].

\subsection{Mathematical statement of the problem}

As previously said, the aim of the first level optimisation is the weight minimisation of the sandwich panel satisfying, simultaneously, constraints of different nature. The design variables (both geometrical and mechanical) of the problem can be grouped into the following vector: 
$\mathbf{x}=\left\{\vartheta, t_{c}, l_{2}, l_{1}, h_{c},\left(R_{0 K}^{A^{*}}\right)_{t},\left(R_{1}^{A^{*}}\right)_{t},\left(\Phi_{1}^{A^{*}}\right)_{t}, h_{t},\left(R_{0 K}^{A^{*}}\right)_{b},\left(R_{1}^{A^{*}}\right)_{b},\left(\Phi_{1}^{A^{*}}\right)_{b}, h_{b}\right\}$

Therefore the optimisation problem can be formulated as follows: $\min _{\mathbf{x}} W(\mathbf{x})$

subject to :

$\lambda_{\text {ref }}-\lambda(\mathbf{x}) \leqslant 0$,

$g_{i}(\mathbf{x}) \leqslant 0$, with $i=1,2, \ldots, 14$.

where $W$ is the weight of the sandwich plate, while $\lambda$ is the first buckling load. $\lambda_{\text {ref }}$ is the buckling load determined on a reference structure having the same in-plane dimensions and boundary conditions than those of the sandwich plate that will be optimised, see Section 6. Constraints $g_{1}(\mathbf{x})$ and $g_{2}(\mathbf{x})$ impose the maximum admissible aspect-ratio between the thickness of the core and each skin, see Eq. (1), whilst constraints from $g_{3}(\mathbf{x})$ to $g_{12}(\mathbf{x})$ (see Eq. (2)) are imposed in order to ensure the positive definiteness of the stiffness tensor of the core. Finally, constraints $g_{13}(\mathbf{x})$ and $g_{14}(\mathbf{x})$ are the geometrical and feasibility constraints imposed on the polar parameters of top and bottom skins, see Eq. (11).

\subsection{Numerical strategy}

Problem (13) is a non-linear, non-convex problem in terms of both geometrical and mechanical variables. Its non-linearity and non-convexity is due on one side on the nature of the objective function and on the other side on the optimisation constraints, specially the constraint on the buckling load that is a high non-convex function in terms of both the orthotropy orientation (bottom and top laminates) and the corrugation angle of the unit cell of the core. In addition, the complexity of such a problem is also due to the existence constraints imposed on the technical moduli of the honeycomb core, see. Eq. (2), that are highly non-convex functions of the geometrical parameter of the unit cell (see Part I). The total number of design variables is 13 while the total number of optimisation constraints is 15 (see Eqs. (12) and (13), respectively).

For the resolution of problem (13) we used the new version of the GA BIANCA $[19,29]$ coupled with both the meso-scale FE model for the numerical homogenisation of the honeycomb core and the macro-scale FE model of the sandwich panel for the buckling analysis of the structure, see Fig. 3. The new version of the GA BIANCA was already successfully applied to solve different kinds of realworld engineering problems, see for example [20,21,30-32,27].

As shown in Fig. 3, for each individual at each generation, we perform a numerical simulation for the evaluation of the effective material properties of the core and a subsequent numerical evaluation of the first buckling load of the sandwich structure along with its weight. The meso-scale FE model uses the geometrical parameters of the unit cell, given by the GA BIANCA, in order to homogenise the honeycomb core and to determine its effective material properties. Afterwards, the macro-scale FE model uses the geometrical and mechanical design variables of the skins given by BIANCA together with the effective material properties of the core (issued from the meso-scale FE model of the cell) to evaluate the first buckling load of the structure and its weight. Therefore, for these purposes the GA BIANCA has been interfaced with the commercial FE code ANSYS ${ }^{\infty}$. The GA BIANCA elaborates the results of the two FE analyses in order to execute the genetic operations. These operations are repeated until the GA BIANCA meets the user-defined convergence criterion.

The generic individual of the GA BIANCA represents a potential solution for the problem at hand. The genotype of the individual for problem (13) is characterised by only one chromosome composed by 13 genes, each one coding a component of the vector of the design variables, see Eq. (12).

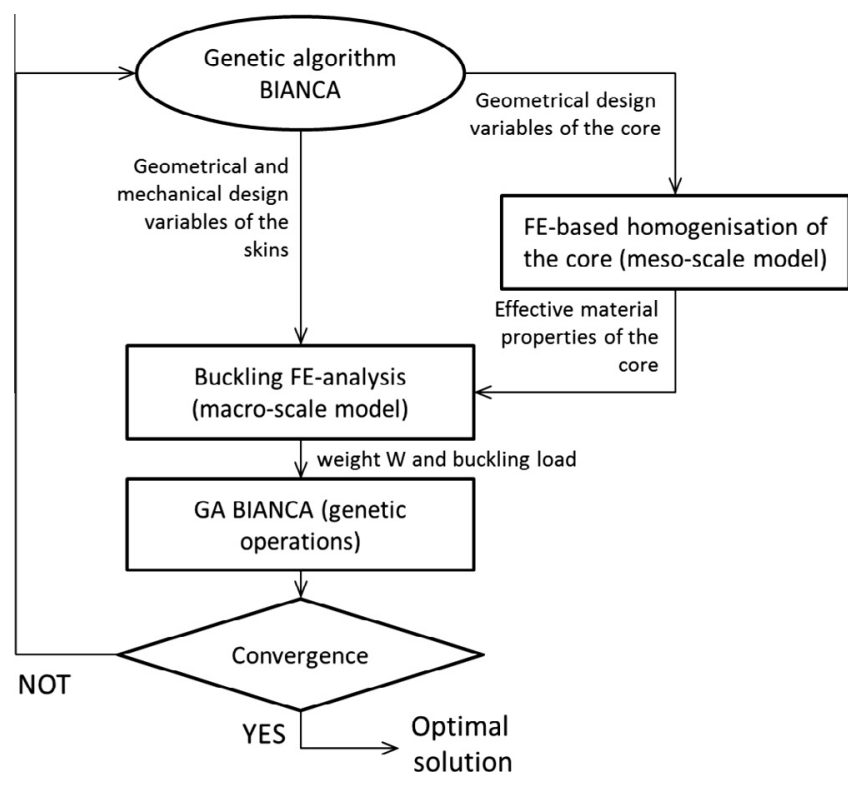

Fig. 3. Logical flow of the numerical procedure for the solution search of the fistlevel problem.

\section{Mathematical formulation of the second-level problem}

The second-level problem concerns the lay-up design of both top and bottom skins. Such a problem consists in determining at least one stacking sequence satisfying the optimum values of both geometric and polar parameters resulting from the first level of the strategy and having the elastic symmetries imposed on the laminate within the formulation of the first-level problem, i.e. quasi-homogeneity and orthotropy. In the framework of the polar formalism, this problem can be stated in the form of an unconstrained minimisation problem:

$\min _{\delta} I\left(f_{i}(\boldsymbol{\delta})\right)$

with

$I\left(f_{i}(\boldsymbol{\delta})\right)=\sum_{i=1}^{6} f_{i}(\boldsymbol{\delta})$

where $\delta$ is the vector of the layers orientations, i.e. the design variables of this phase, while $f_{i}(\boldsymbol{\delta})$ are quadratic functions in the space of polar parameters, each one representing a requirement to be satisfied, such as orthotropy, uncoupling, etc. For the problem at hand we have:

$\left.f_{1}(\boldsymbol{\delta})=\frac{\left|\Phi_{0}^{A^{*}}(\boldsymbol{\delta})-\Phi_{1}^{A^{*}}(\boldsymbol{\delta})\right|}{\pi / 4}-K^{A^{*}(o p t)}\right)^{2}$,

$\left.f_{2}(\boldsymbol{\delta})=\frac{R_{0}^{A^{*}}(\boldsymbol{\delta})-R_{0}^{A^{*}(o p t)}}{R_{0}}\right)^{2}$

$\left.f_{3}(\boldsymbol{\delta})=\frac{R_{1}^{A^{*}}(\boldsymbol{\delta})-R_{1}^{A^{*}(o p t)}}{R_{1}}\right)^{2}$,

$\left.f_{4}(\boldsymbol{\delta})=\frac{\left|\Phi_{1}^{A^{*}}(\boldsymbol{\delta})-\Phi_{1}^{A^{*}(o p t)}\right|}{\pi / 4}\right)^{2}$,

$f_{5}(\boldsymbol{\delta})=\left(\frac{\|\mathbf{C}(\boldsymbol{\delta})\|}{\|\mathbf{Q}\|}\right)^{2}$

$f_{6}(\boldsymbol{\delta})=\left(\frac{\left\|\mathbf{B}^{*}(\boldsymbol{\delta})\right\|}{\|\mathbf{Q}\|}\right)^{2}$, 


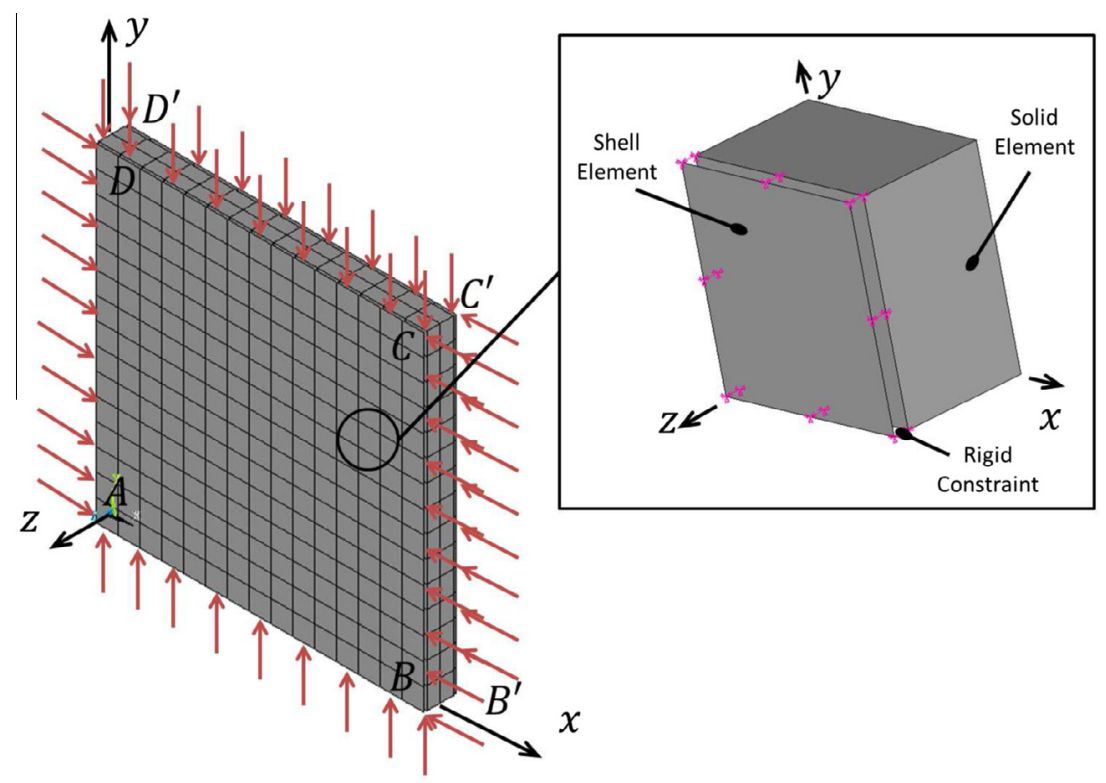

Fig. 4. Mesh and rigid constraint equations for the FE model of the sandwich panel.

where $f_{1}(\boldsymbol{\delta})$ represents the elastic requirement on the orthotropy of the laminate having the prescribed shape (imposed by the value of $K^{A^{*}}$ provided by the first step of the procedure), $f_{2}(\boldsymbol{\delta}), f_{3}(\boldsymbol{\delta})$ and $f_{4}(\boldsymbol{\delta})$ are the requirements related to the prescribed values of the optimal polar parameters issued from the first-level problem, while $f_{5}(\boldsymbol{\delta})$ and $f_{6}(\boldsymbol{\delta})$ are linked to the quasi-homogeneity condition.

$I\left(f_{i}(\boldsymbol{\delta})\right)$ is a positive semi-definite convex function in the space of laminate polar parameters, since it is defined as a sum of convex functions, see Eqs. (15) and (16). Nevertheless, such a function is highly non-convex in the space of plies orientations because the laminate polar parameters depend upon circular functions of layers orientation angles, see Eq. (8). Moreover, one of the advantages of such a formulation consists in the fact that the absolute minima of $I\left(f_{i}(\boldsymbol{\delta})\right)$ are known a priori since they are the zeroes of this function. For more details about the nature of the second-level problem see $[19,21,24]$. We used the GA BIANCA to find a solution also for the second-level problem. In this case, each individual has a genotype composed of $n$ chromosomes, one for each ply, characterised by a single gene coding the layer orientation. It must be pointed out that problem (14) must be solved two times, i.e. for each laminated plate composing the panel (bottom and top faces). We recall that the optimal thickness of the two laminated skins is a result of the first step of the procedure, where the thickness variables $h_{t}$ and $h_{b}$ are set as discrete variables with a discretisation step equal to the thickness of the elementary ply, see Table 2. From the knowledge of $h_{t}$ and $h_{b}$ we can, hence, determine the optimal number of plies composing each skin.

As conclusive remark of this section, we want to highlight the fact that each ply orientation can get all the values in the range $\left[-89^{\circ}, 90^{\circ}\right]$ with a discretisation step of $1^{\circ}$. Such a discretisation step has been chosen in order to prove that laminates with given elastic properties (such as membrane/bending uncoupling, membrane orthotropy, etc.) can be obtained by abandoning the wellknown conventional rules for tailoring the laminate stack (e.g. symmetric-balanced stacks) which extremely shrink the search space for the problem at hand. The true advantages in using "non-conventional" staking sequences consist in the fact that on one hand with a discretisation step of one degree for the plies orientations we can explore the overall design space of problem (14) and on the other hand we can find very general stacks (nor symmetric neither balanced) that fully meet the elastic properties resulting from the first step of the procedure with a fewer number of plies (hence lighter) than the standard stacks, see [19,21].

\section{Finite element models of the sandwich plate}

The FE models used at the first-level of the strategy are built using the FE commercial code ANSYS ${ }^{\circledR}$. The FE analyses are conducted to determine the value of the objective and constraint functions for each individual, i.e. for each point in the design space, at the current generation.

The need to analyse, within the same generation, different geometrical configurations (plates with different geometrical and material properties), each one corresponding to an individual, requires the creation of an ad-hoc input file for the FE code that has to be interfaced with BIANCA. The FE model must be conceived to take into account a variable geometry, material and mesh. Indeed, for each individual at the current generation the FE code has to be able to vary in the correct way the number of elements wherein the structure is discretised, thus a correct parametrisation of the model has to be achieved.

During the optimisation process of the first level of the strategy we have to perform, for each individual, seven FE analyses (see Fig. 3): six static analyses on the FE model of the unit cell of the honeycomb core (in order to determine the effective material properties, see also Part I) and a linear buckling analysis of the whole sandwich panel.

\subsection{Finite element model of the unit cell}

In order to accurately determine the effective properties of the core we need a numerical homogenisation phase. In this way the periodic honeycomb structure is replaced, at the macro-scale, by an equivalent orthotropic homogeneous solid whose material properties depend on the geometric parameters of the repetitive unit of the honeycomb. In particular, these properties have been determined using the strain energy homogenisation technique of periodic media, as described in Part I of the present work. This technique makes use of the repetitive unit of the periodic structure to approximate its effective properties at the macro-scale level. As 
Table 3

BCs of the FE model of the sandwich panel.

\begin{tabular}{ll}
\hline Sides & Constraint \\
\hline $\mathrm{AB}, \mathrm{A}^{\prime} \mathrm{B}^{\prime}, \mathrm{CD}, \mathrm{C}^{\prime} \mathrm{D}^{\prime}$ & $U_{x}=0$ \\
& $U_{z}=0$ \\
$\mathrm{BC}, \mathrm{B}^{\prime} \mathrm{C}^{\prime}, \mathrm{DA}, \mathrm{D}^{\prime} \mathrm{A}^{\prime}$ & $U_{y}=0$ \\
& $U_{z}=0$ \\
\hline
\end{tabular}

Table 4

Reference solution for the sandwich panel design problem.

\begin{tabular}{ll}
\hline$a, b[\mathrm{~mm}]$ & 1500.00 \\
$\vartheta[\mathrm{deg}]$ & 60.00 \\
$t_{c}[\mathrm{~mm}] \times 10^{-2}$ & 6.35 \\
$l_{2}[\mathrm{~mm}] \times 10^{-1}$ & 18.33 \\
$l_{1}[\mathrm{~mm}] \times 10^{-1}$ & 36.66 \\
$h_{c}[\mathrm{~mm}]$ & 80 \\
$\left(R_{0 K}^{A^{*}}\right)_{t}[\mathrm{MPa}]$ & -9855.21 \\
$\left(R_{1}^{A^{*}}\right)_{t}[\mathrm{MPa}]$ & 5358.28 \\
$\left(\Phi_{1}^{A^{*}}\right)_{t}[\mathrm{deg}]$ & 0.0 \\
$h_{t}[\mathrm{~mm}]$ & 4.00 \\
$\left(R_{0 K}^{A^{*}}\right)_{b}[\mathrm{MPa}]$ & -9855.21 \\
$\left(R_{1}^{A^{*}}\right)_{b}[\mathrm{MPa}]$ & 5358.28 \\
$\left(\Phi_{1}^{A^{*}}\right)_{b}[\mathrm{deg}]$ & 0.0 \\
$h_{b}[\mathrm{~mm}]$ & 4.00 \\
Skins Weight $[\mathrm{kg}]$ & 28.44 \\
Core weight $[\mathrm{kg}]$ & 12.58 \\
Panel weight $[\mathrm{kg}]$ & 41.02 \\
Buckling load $[\mathrm{N} / \mathrm{mm}]$ & 5691.88 \\
Stacking sequence & No. of \\
\hline $45 / 0 / 45 / 45 /-45 / 45 /-45 / 0 / 0 / 45 /-45 / 45 /-45 /-45 / 0 / 45]_{s}$ & 32 \\
\hline
\end{tabular}

illustrated in Fig. 4 of Part I the meso-scale FE model is built using the 20-node ANSYS solid element SOLID186. For a deeper insight in the matter the reader is addressed to Part I of this study.

\subsection{Finite element model of the sandwich panel}

At the macro-scale the structure is modelled with a combination of shell and solid elements. In particular, the laminated skins are modelled using ANSYS SHELL281 elements with 8-nodes and six degrees of freedom (DOFs) per node, and their mechanical behaviour is described by defining directly the normalised stiffness tensors $\mathbf{A}^{*}, \mathbf{B}^{*}$ and $\mathbf{D}^{*}$. The equivalent solid representing the core is modelled using ANSYS SOLID186 elements with 20-nodes and 3 DOFs per node having the material properties calculated via the six FE static analyses of the unit cell. Concerning the BCs of the FE model at the macro-scale, they are depicted in Fig. 4 and listed in Table 3. In particular, such BCs are applied on the sides of the top and bottom skins and not on the core.

The compatibility of the displacement field between skins (modelled with shell elements) and core (modelled with solid elements) is achieved by using ANSYS CERIG rigid constraints (also called rigid links) whose formulation is based upon a classical master-slave scheme, see [33] for more details. Rigid constraints are imposed on each node belonging to contiguous solid and shell elements as depicted in Fig. 4. In particular, rigid links are defined between the nodes of the middle plane of the top (bottom) skin and the corresponding ones of the top (bottom) surface of the solid core. In this case the master nodes are those belonging to shell elements (the skins), while slave nodes are those belonging the top and bottom surfaces of the core.
Table 5

Genetic parameters of the GA BIANCA for both first and second-level problems.

\begin{tabular}{lll}
\hline Genetic parameters & & \\
\hline & 1st level problem & 2nd level problem \\
\hline$N_{\text {pop }}$ & 1 & 1 \\
$N_{\text {ind }}$ & 160 & 500 \\
$N_{\text {gen }}$ & 200 & 500 \\
$p_{\text {cross }}$ & 0.85 & 0.85 \\
$p_{\text {mut }}$ & $1 / N_{\text {ind }}$ & $1 / N_{\text {ind }}$ \\
Selection & Roulette-wheel & Roulette-wheel \\
Elitism & Active & Active
\end{tabular}

Finally, before starting the optimisation process we conducted a sensitivity study (not reported here for the sake of brevity) on the proposed FE model with respect to the mesh size. We checked that a mesh having 12088 DOFs, i.e. showing two divisions through the core thickness $h_{c}$, is sufficient to properly evaluate the first buckling load of the structure.

\section{Studied cases and results}

The optimisation strategy has been applied to the sandwich structure depicted in Fig. 1. As previously said, the sandwich plate is composed by two laminated skins and a honeycomb core. We remind that the skins are made of carbon-epoxy unidirectional orthotropic laminae while the honeycomb core is made of aluminium foils with the material properties listed in Table 1.

In order to show the effectiveness of the proposed approach we studied two different cases. In the first case we perform the optimal design of a sandwich plate having a fixed core thickness, while in the second case we introduce also the core thickness among the design variables. Moreover, for each case we considered two subcases: the first one wherein we design a sandwich plate with identical skins and the second one, more general, where the sandwich plate is characterised by different skins. It should be pointed out that the 1st studied case is considered in order to show that our strategy is capable to search for an optimal solution more efficient (in terms of weight and first buckling load) than the reference one, even when the design problem expects a design constraint on the overall dimensions of the structure, for example on the thickness of the core. On the other hand, with the 2nd studied case our aim is to prove that the optimisation strategy is able to find an optimum configuration of the sandwich plate with better overall properties than the reference structure in the most general case, i.e. when the thickness of the core is included among the design variables.

As said in Section 3 the goal of this optimisation problem is to minimise the weight of the sandwich panel satisfying simultaneously several constraints, in particular, a constraint on the first buckling load that has to be greater or equal than the first buckling load of a reference structure having the characteristics listed in Table 4. In particular, the reference structure has identical skins (hence it is a symmetric panel) each one composed by 32 plies with the stacking sequence listed in Table 4 . We have chosen as reference solution a non-trivial configuration with a honeycomb core characterised by a unit cell having the typical dimensions of commercial honeycombs (a regular hexagonal cell whose sizes are taken from [34,35]) and two very stiff skins. In fact, the weight and the stiffness properties (in terms of buckling load) of such a reference configuration are typical of real-world engineering applications (in other words the reference solution still represents a "good" compromise between weight and stiffness requirements).

Concerning the BCs we remind that we considered, for every studied case, a simply supported panel submitted to bi-axial compressive loads $\left(N_{x}=N_{y}\right)$ applied along the edges of top and bottom skins, see Fig. 4 and Table 3 . 


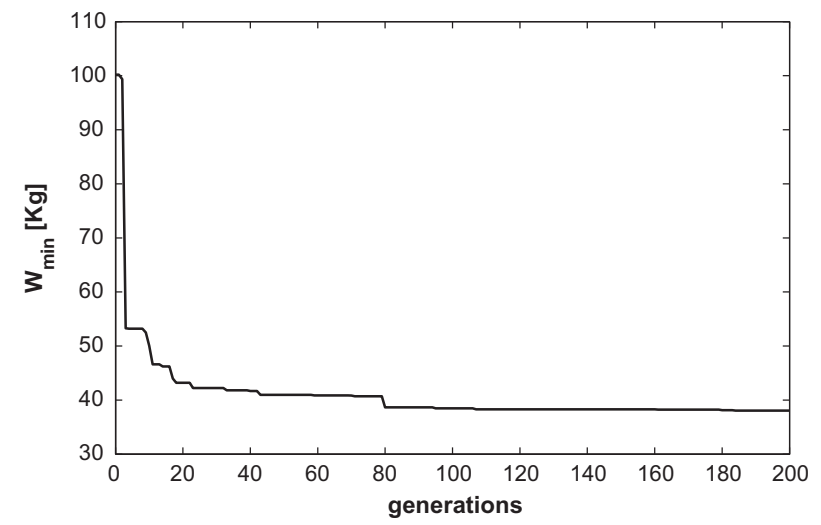

Fig. 5. Best values of the objective function along generations, case 1.a.

Regarding the setting of the genetic parameters for the GA BIANCA used to solve both first and second-level problems they are listed in Table 5. Moreover, concerning the constraint-handling technique for the first-level problem the Automatic Dynamic Penalization (ADP) method has been employed, see [29]. For more details on the numerical techniques developed within the new version of BIANCA and the meaning of the values of the different parameters tuning the GA the reader is addressed to [19,21].

\subsection{Case 1.a: sandwich plate with fixed core thickness and identical} skins

For this first example, since the height of the core is fixed $a$ priori $\left(h_{c}=80 \mathrm{~mm}\right.$ ) and the panel is characterised by identical skins, the number of design variables reduces from 13 to 8 . Moreover, the fact that top and bottom skins are assumed to be identical implies the reduction of optimisation constraints: they pass from 15 to 13 because constraints $g_{1}(\mathbf{x})$ and $g_{2}(\mathbf{x})$ (Eq. (2)) as well as $g_{13}(\mathbf{x})$ and $g_{14}(\mathbf{x})$ (Eq. (11)) are coincident.

The optimal values of the geometric as well as mechanical design variables resulting from the first-level of the optimisation strategy are listed in Table 6. The global constrained minimum has been found by BIANCA after 80 generations, as shown in Fig. 5. As it can be easily seen, the optimum configuration has a weight of $38.04 \mathrm{~kg}$ (about 7\% lower than that of the reference structure) with a first buckling load of $5695.00 \mathrm{~N} / \mathrm{mm}$ (almost equal to the reference one).

We want to highlight the following aspects which naturally arises when analysing the optimum configuration of the system provided by the first step of the procedure:

1. through this first case-study, which is the most limited in terms of span of the search space (fixed thickness of the core and identical skins), we have shown the possibility of conceiving an optimal solution of sandwich panel lighter than the reference one but having the same overall dimensions and the same stiffness. In particular, the weight reduction is linked only to the variation of the geometry of the unit cell (the weight contribution of the skins is the same as that of the reference panel) whereas the constraint on the buckling load is satisfied thanks to the optimal combination of the polar parameters characterising the laminated skins;

2. when looking at the values of Tables 4 and 6 , one can notice that the reference solution is characterised by an orthotropy shape with $K^{A^{*}}=1$, whilst the optimum configuration has the orthotropy shape with $K^{A^{*}}=0$. This implies that the nature of the laminate stack (that we will determine in the second level of the strategy) will be completely different.

As a consequence of these considerations, we can suppose that a further generalisation of the optimisation problem, i.e. by considering different skins (as we do in the subsequent case 1.b), should lead to an optimal solution lighter than that obtained in this first case study.

We have to consider now the second-level problem: the design of the laminate lay-up. Since in this case the laminates of the skins are identical, the second-level problem is solved only one time. Table 7 shows the best stacking sequences for all the studied cases. As in each numerical technique, the quality of solutions found by BIANCA can be estimated on the basis of a numerical tolerance, that is the residual. For a discussion on the importance of the numerical residual in problems of this type, the reader is addressed to $[19,24] . I\left(f_{i}(\boldsymbol{\delta})\right)$ is a non-dimensional function, thus the residual of the solution is a non-dimensional quantity too. The residual in the last column of Table 7 is the value of the global objective function $I\left(f_{i}(\boldsymbol{\delta})\right)$ for the solution indicated aside (we remind that exact solutions correspond to the zeroes of the objective function, see [24]). From Table 7 we can see that the optimal stacks (for all

Table 6

Numerical results of the fist-level optimisation problem for both 1st and 2nd cases.

\begin{tabular}{|c|c|c|c|c|}
\hline & Solution case $1 . \mathrm{a}$ & Solution case $1 . b$ & Solution case $2 . \mathrm{a}$ & Solution case $2 . \mathrm{b}$ \\
\hline$\vartheta[\mathrm{deg}]$ & 48.00 & 47.00 & 44.00 & 49.00 \\
\hline$t_{c}[\mathrm{~mm}] \times 10^{-2}$ & 5.40 & 6.50 & 6.60 & 4.40 \\
\hline$l_{2}[\mathrm{~mm}] \times 10^{-1}$ & 6.10 & 3.00 & 3.70 & 5.50 \\
\hline$l_{1}[\mathrm{~mm}] \times 10^{-1}$ & 49.00 & 49.00 & 85.00 & 57.00 \\
\hline$h_{c}[\mathrm{~mm}]$ & 80 & 80 & 121.00 & 121.00 \\
\hline$\left(R_{0 K}^{A^{+}}\right)_{t}[\mathrm{MPa}]$ & 19324.70 & 19594.40 & 13429.00 & -18785.20 \\
\hline$\left(R_{1}^{A^{*}}\right)_{t}[\mathrm{MPa}]$ & 2053.21 & 356.17 & 5677.75 & 1822.75 \\
\hline$\left(\Phi_{1}^{A^{*}}\right)_{t}[\mathrm{deg}]$ & 45.00 & -45.00 & -60.00 & 0.00 \\
\hline$h_{t}[\mathrm{~mm}]$ & 4.00 & 3.50 & 3.00 & 3.00 \\
\hline$\left(R_{0 K}^{A^{*}}\right)_{b}[\mathrm{MPa}]$ & 19324.70 & 19324.70 & 13429.00 & 9267.36 \\
\hline$\left(R_{1}^{A^{*}}\right)_{b}[\mathrm{MPa}]$ & 2053.21 & 167.61 & 5677.75 & 1948.45 \\
\hline$\left(\Phi_{1}^{A^{*}}\right)_{b}[\mathrm{deg}]$ & 45.00 & 45.00 & -60.00 & -45.00 \\
\hline$h_{b}[\mathrm{~mm}]$ & 4.00 & 3.5 & 3.00 & 3.00 \\
\hline Skins Weight [kg] & 28.44 & 24.88 & 21.33 & 21.33 \\
\hline Core weight [kg] & 9.60 & 12.00 & 10.91 & 10.41 \\
\hline Panel weight $[\mathrm{kg}]$ & 38.04 & 36.88 & 32.24 & 31.74 \\
\hline Buckling load $[\mathrm{N} / \mathrm{mm}]$ & 5695.00 & 5704.32 & 5700.26 & 5721.29 \\
\hline
\end{tabular}


cases) are very general stacks which completely satisfy the elastic requirements of the laminate. In fact, for this first case Fig. 6 shows the first component of the homogenised stiffness tensors of the laminate, i.e. $\mathbf{A}^{*}, \mathbf{B}^{*}$ and $\mathbf{D}^{*}$ : the solid line refers to the extension tensor, the dashed one to the bending tensor, while the dash-dotted one is linked to the coupling stiffness tensor. We can see that the laminate is uncoupled as the dash-dotted curve is reduced to a point in the center of the plot $\left(B_{11}\right.$ is practically null), homogeneous as the solid and dashed curves are almost coincident and orthotropic because there are two orthogonal axes of symmetry in the plane. In addition, the main orthotropy axis for this case is oriented at $\Phi_{1}^{A^{*}}=45^{\circ}$ as indicated in Table 6 .

6.2. Case 1.b: sandwich plate with fixed core thickness and different skins

In this case the height of the core is still fixed a priori $\left(h_{c}=80\right.$ $\mathrm{mm}$ ) while the panel is characterised by different skins, hence, with respect to case 1.a the number of design variables increases from 8 to 12, while the optimisation constraints increase from 13 to 15 and correspond to those of Eq. (13).

The optimal values of geometric as well as mechanical design variables resulting from the first level of the optimisation strategy are listed in Table 6. The global constrained minimum has been found by BIANCA after 180 generations as depicted in Fig. 7 . The optimum configuration weighs $36.88 \mathrm{~kg}$ (a reduction of $10 \%$ when compared to that of the reference structure) with a first buckling load of $5704.32 \mathrm{~N} / \mathrm{mm}(0.2 \%$ greater than the reference one).

This solution, as expected, is lighter than that of the case 1.a with a difference of $1.16 \mathrm{~kg}$ with almost the same buckling load. This difference is mainly due to the weight contribution given by the two skins. In fact, the optimal solution of the present case is characterised by two different skins with a thickness of $3.5 \mathrm{~mm}$ (28 plies), i.e. a reduction of four layers when compared to the thickness of the skins of case 1.a.

On the other side, the thickness reduction of the skins has led to a thickness increment of the cell walls of the core and, therefore, to an increase of its weight of about $2.4 \mathrm{~kg}$ compared to the solution 1.a. The variation of the geometry of the unit cell together with the variation of the polar parameters of the skins occur in order to meet the prescribed minimal stiffness of the whole structure through the constraint on the first buckling load.

Finally, the rise in the number of variables involved in the first-level problem has led the GA to find an optimal solution less bulky (thinner than the reference one of about $1 \mathrm{~mm}$ ) and lighter of $4.14 \mathrm{~kg}$ compared to the reference solution and of $1.16 \mathrm{~kg}$ compared to the optimal solution of case 1.a.

Concerning the second-level problem, since in this case the laminated skins are different, the laminate design problem must be solved separately for top and bottom skins. Table 7 shows the best stacking sequences for both the skins for the present case, while Fig. 8 shows the polar diagrams for the first component of the corresponding homogenised stiffness tensor. Regarding the nature of the optimal stacks, even for this case, we can repeat the same considerations as those of case 1.a.

\subsection{Case 2.a: sandwich plate with variable core thickness and identical skins}

The aim of cases 2.a and 2.b consists in proving that more efficient configurations of the sandwich panel can be found when considering the most general case, i.e. by including the core height $h_{c}$ among the design variables of the problem. 


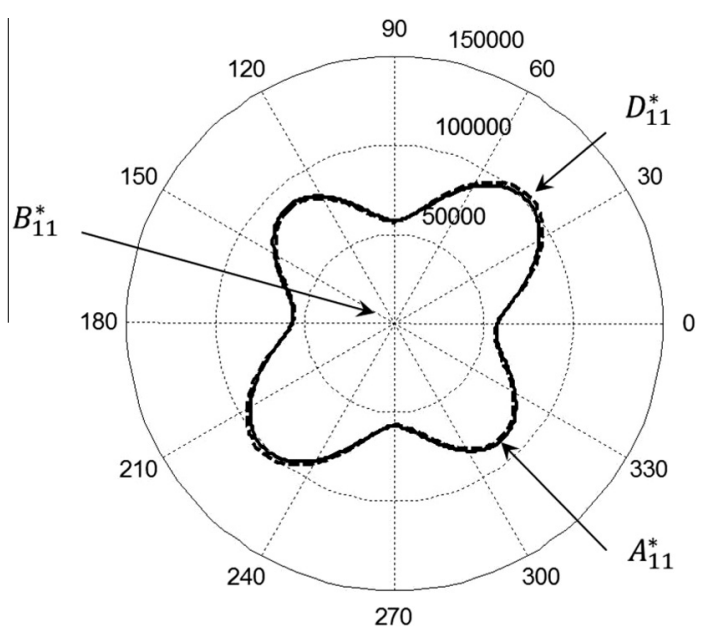

Fig. 6. First component of the homogenised stiffness tensors of the laminate [MPa], case 1.a.

In this first sub-case we consider a panel with identical skins. As in the case 1.a, this implies a reduction of the number of design variables that passes from 13 to 9 when compared to the most general case, whilst the number of optimisation constraints reduces from 15 to 13 .

The optimal values of geometric as well as mechanical design variables resulting from the first-level of the optimisation strategy are listed in Table 6. The global constrained minimum has been found by BIANCA after 170 generations as illustrated in Fig. 9. The optimum configuration has a weight of $32.24 \mathrm{~kg}$ (about $21.4 \%$ lower than that of the reference structure) with a first buckling load of $5700.26 \mathrm{~N} / \mathrm{mm}$ (almost equal to the reference one).

As provided in the comments to case 1.a, the generalisation of the optimisation problem has led to more efficient solutions, i.e. lighter solutions. In this case, both skins and core are lighter than those of solution 1.b: the skins are lighter than those of the reference solution of $3.55 \mathrm{~kg}$, whilst the core is lighter than its reference counterpart of about $1.09 \mathrm{~kg}$, see Table 6 . In particular the skins, for the present solution, are composed by 24 plies $\left(h_{t}=h_{b}=3 \mathrm{~mm}\right)$. This weight reduction is translated, in terms of geometrical characteristics of the structure, in a core thickness incrementation (that passes from $80.00 \mathrm{~mm}$ for cases 1.a and 1.b to $121.00 \mathrm{~mm}$ for the present case) in order to meet the constraint on the first buckling load and, thus spacing the skins in order to increase the bending stiffness of the panel.

Concerning the results of the second-level problem the optimal stack is listed in Table 7 while the related polar diagrams are

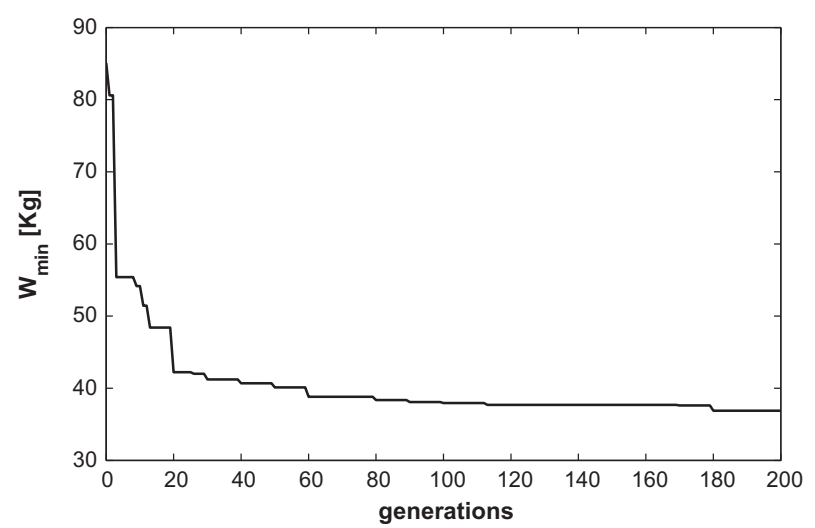

Fig. 7. Best values of the objective function along generations, case 1.b.
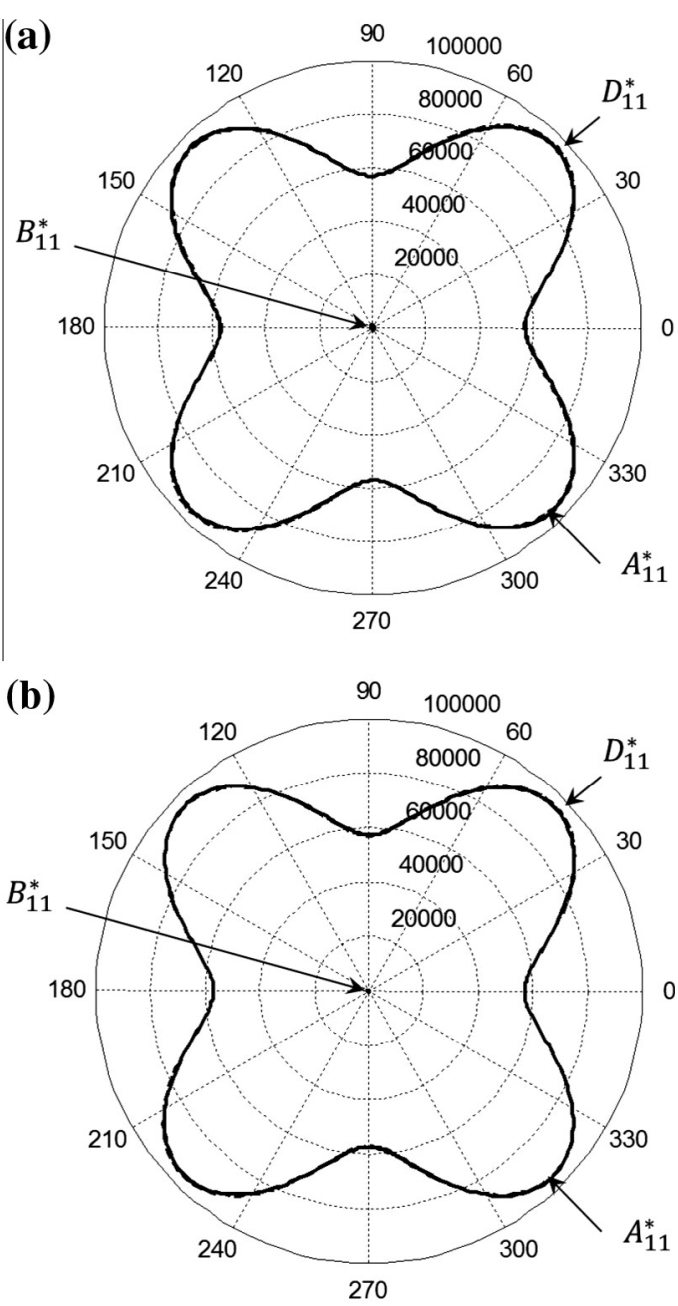

Fig. 8. First component of the homogenised stiffness tensors of the laminate [MPa], (a) top skin and (b) bottom skin, case 1.b.

depicted in Fig. 10. The considerations already done for the previous cases can be repeated verbatim for the present one.

6.4. Case 2.b: sandwich plate with variable core thickness and different skins

In this last example we consider the most general case where the thickness of the core is included among the design variables

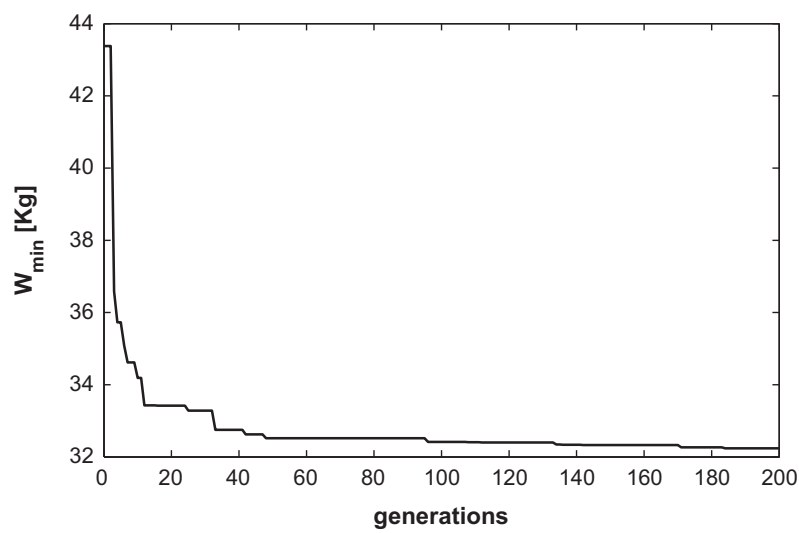

Fig. 9. Best values of the objective function along generations, case 2.a. 


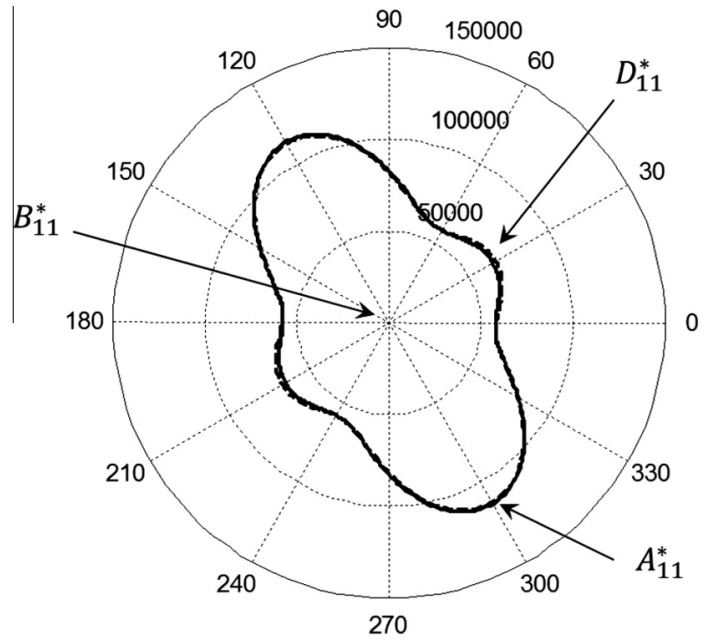

Fig. 10. First component of the homogenised stiffness tensors of the laminate [MPa], case 2.a.

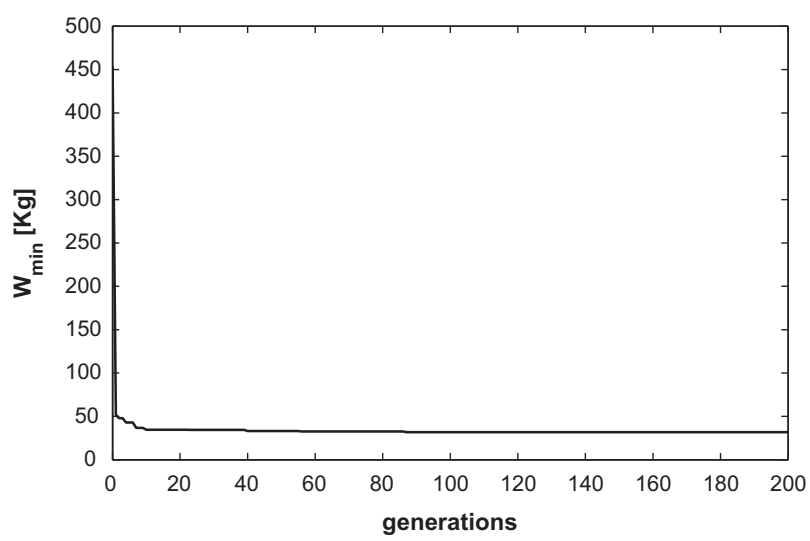

Fig. 11. Best values of the objective function along generations, case 2.b.

and the skins are different, thus the vector of design variables corresponds to that of Eq. (12).

The optimal values of geometric as well as mechanical design variables resulting from the first level of the optimisation strategy are listed in the last column of Table 6. The global constrained minimum has been found by BIANCA after 90 generations as shown in Fig. 11. The optimum configuration has a weight of $31.74 \mathrm{~kg}$ (about $22.6 \%$ lower than that of the reference structure) with a first buckling load of $5721.29 \mathrm{~N} / \mathrm{mm}$ ( $0.5 \%$ greater than the reference one).

The solution given by this last case is the lightest one and also the stiffest one: this is due to the combined action on one hand of the core height that increases up to $121 \mathrm{~mm}$, thus spacing the skins and increasing the flexural stiffness of the sandwich panel and on the other hand of the polar parameters of the skins (that are different) which attain the optimum configuration that slightly increases the buckling load. It can be noticed that, unlike case 1.b, here the skins are different not only in terms of the polar parameters values but also in terms of the shape of orthotropy, see Table 6 : the top skin has a negative value of the parameter $R_{0 K}^{A^{*}}$ (orthotropy shape with $K^{A^{*}}=1$ ), while the bottom skin is characterised by an orthotropy shape with $K^{A^{*}}=0$. For the rest, the overall dimensions are identical to those of the solution of case 2.a, whilst at the meso-scale the unit cell geometry differs from that of the solution of case 2 .a.
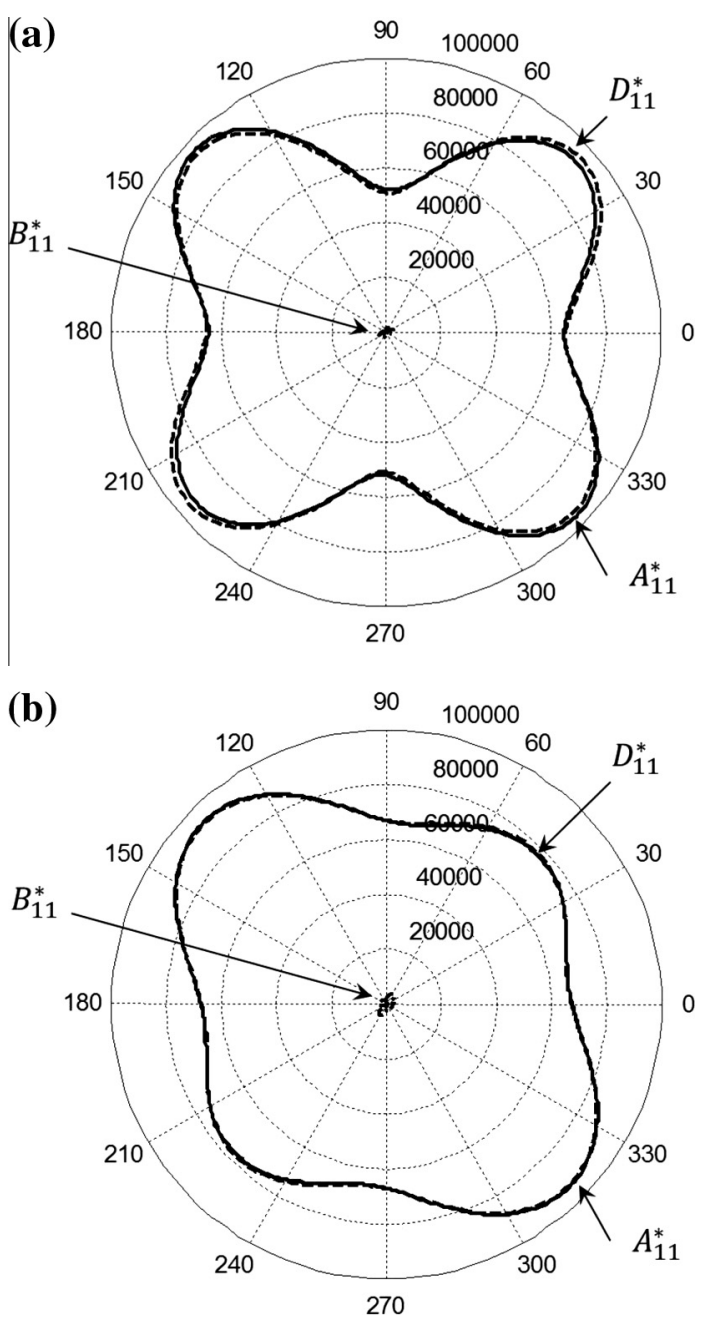

Fig. 12. First component of the homogenised stiffness tensors of the laminate [MPa], (a) top skin and (b) bottom skin, case 2.b.

Concerning the results of the second-level problem the optimal stacks for both bottom and top skins are listed in Table 7, while the related polar diagrams are depicted in Fig. 12. We want to remark the fundamental difference in the orthotropy shape of the laminated skins: as it can be easily seen from Fig. 12 the orthotropy shape of the top skin, which has the main orthotropy axis oriented at $0^{\circ}$, is the so-called "dog bone" orthotropy (due to the shape of the polar diagram), whilst the orthotropy shape of the bottom skin is a standard shape with the orthotropy axes oriented at $-45^{\circ}$, see also Table 6. For the rest, the considerations already done for case 2.a can be repeated here.

\section{Conclusions}

The design strategy presented in this paper is a numerical optimisation procedure characterised by several features that make it an innovative, effective and general method for the design of complex multi-scale structures. In the present work this strategy has been employed to deal with the problem of the optimum design of a sandwich panel composed of two laminated skins and a honeycomb core.

On one hand, the design process is not submitted to restrictions: any parameter characterising our structure is an 
optimisation variable. This allows us to look for a true global minimum, hard to be obtained otherwise.

On the other hand, in order to solve the problem in a very general way we split it into two distinct but linked non-linear minimisation problems which are solved within the same multiscale procedure developed on two different levels. The first level of the procedure involves two different scales: the macro-scale of the sandwich panel composed by two homogeneous anisotropic plates (the skins) along with an homogeneous anisotropic core and the meso-scale of the honeycomb core modelled through its representative volume element. Many types of design variables are involved within this first level: the geometrical parameters of the honeycomb unit cell (meso-scale) together with the overall thickness and the laminate polar parameters of each skin (macro-scale). The second level of the procedure concerns the meso-scale of the laminated skins: in this phase, we look for the optimal stacking sequences giving the optimum value of the thickness and of the laminate polar parameters provided by the first step. Concerning the numerical computations, they are carried out by a genetic algorithm, BIANCA, able to handle both continuous and discrete-valued variables during the same iterations as well as the constraints of the problem. For the first-level problem, the code BIANCA is interfaced with the FE code ANSYS that invokes seven different FE analyses in order to compute the objective as well as the constraint functions of the problem.

In addition, the mechanical characteristics of the laminated plates are represented by the polar formalism, that gives several advantages, namely to explicit elastic symmetries, elastic and geometric bounds, and to eliminate from the procedure redundant mechanical properties. In addition, the use of the polar formalism leads us to easily formulate the second-level problem which takes into account any combination of requirements on elastic properties.

To our best knowledge, this is the first time that the problem of the least-weight design of a sandwich panel with a honeycomb core is formulated in a very general way, i.e. by abandoning the usual simplifying hypotheses and the standard rules, and, hence, by taking into account all geometrical and material parameters characterising the structure as design variables and considering, within the same procedure, two different scales (meso and macro).

Finally, the use of an evolutionary strategy along with the fact that the problem is stated in the most general case, leads us to find some non-conventional configurations more efficient than the standard ones. In fact, with some examples we show that when standard rules for tailoring the laminate stacks are abandoned and all of the parameters characterising the structure, at each scale, are included among the design process a significant weight saving (with respect to a given reference structure) can be obtained: up to $10 \%$ when the core height is kept constant and up to $22 \%$ when the core height is included among the variables.

However, the proposed solutions cannot be, probably, still employed for industrial purposes as they are not manufacturable with the current technological capabilities. Thanks to the general nature and the flexibility of the proposed optimisation strategy we can take into account also manufacturability requirements by introducing further optimisation constraints and/or modifying the design space of the optimisation problem. These considerations remain still valid if the designer wants to include within the process constraints of different nature, e.g. on strength, yielding, delamination, etc. or if he wants to improve the mathematical model to be optimised (i.e. the numerical model simulating the mechanical response of the structure) by introducing the influence of geometrical imperfections, material as well as geometrical non-linearity, etc. All of these aspects can be easily integrated within the optimisation process without altering its overall architecture and they do not represent a limitation to the proposed strategy, on the contrary they could be an interesting challenge for future researches on real-life applications.

\section{References}

[1] Allen HG. Analysis and design of structural sandwich panels. Pergamon Press; 1969.

[2] Huang SN, Alspaught DW. Minimum weight sandwich beam design. AIAA J $1974 ; 12: 1617-8$

[3] Velea MN, Wennhage P, Zenkert D. Multi-objective optimisation of vehicle bodies made of frp sandwich structures. Compos Struct 2014;111: 75-84.

[4] Triantafillou TC, Gibson LJ. Minimum weight design of foam core sandwich panels for a given strength. Mater Sci Eng 1987;95:55-62.

[5] Vinson JR. Plate and panel structures of isotropic, composite and piezoelectric materials, including sandwich construction. Springer; 2005.

[6] Aimmanee S, Vinson JR. Analysis and optimization of foam-reinforced web core composite sandwich panels under in-plane compressive loads. J Sandwich Struct Mater 2002;4:115-39.

[7] Steeves CA. Optimizing sandwich beams for strength and stiffness. J Sandwich Struct Mater 2012;14:573-95.

[8] Dragoni E. Optimal mechanical design of tetrahedral truss cores for sandwich constructions. J Sandwich Struct Mater 2013;15:464-84.

[9] Asadi H, Gorji M, Ashouri D, Khalkhali A, Post buckling modelling and optimization of sandwich panels with corrugated cores. In: 5th WSEAS/ IASME international conference on engineering education, Heraklion, Greece; 2008

[10] Wennhage P. Weight minimization of sandwich panels with acoustic and mechanical constraints. J Sandwich Struct Mater 2001:3:22-49.

[11] Wennhage P. Weight optimization of sandwich panel with acoustic constraints, experimental verification. J Sandwich Struct Mater 2002;4: 353-65.

[12] Wennhage P. Weight minimization of large scale sandwich structures with acoustic and mechanical constraints. J Sandwich Struct Mater 2003;5: 253-66.

[13] Nayak SK, Singh AK, Belegundu AD, Yen CF. Process for design optimization of honeycomb core sandwich panels for blast load mitigation. Struct Multidiscip Optim 2013;47(5):749-63.

[14] Caccese V, Ferguson JR, Edgecomb MA. Optimal design of honeycomb material used to mitigate head impact. Compos Struct 2013;100:404-12.

[15] Qiao P, Fan W, Davalos JF, Zou G. Homogenization and optimization of sinusoidal honeycomb cores for transverse shear stiffness. J Sandwich Struct Mater 2008;10:385-412.

[16] Hudson CW, Carruthers JJ, Robinson AM. Multiple objective optimisation of composite sandwich structures for rail vehicle floor panels. Compos Struct 2010;92:2077-82.

[17] Vannucci P. Plane anisotropy by the polar method. Meccanica 2005;40: 437-54.

[18] Verchery G, Les invariants des tenseurs d'ordre 4 du type de l'élasticité. In: Proc. of colloque Euromech 115, VIllard-de-Lans, (France); 1979.

[19] Montemurro M, Optimal design of advanced engineering modular systems through a new genetic approach [Ph.D. thesis]. UPMC, Paris VI, France. <http:// tel.archives-ouvertes.fr/tel-00955533>; 2012

[20] Montemurro M, Vincenti A, Vannucci P. A two-level procedure for the global optimum design of composite modular structures - application to the design of an aircraft wing. Part 1: theoretical formulation. J Optim Theory Appl 2012:155(1):1-23.

[21] Montemurro M, Vincenti A, Vannucci P. A two-level procedure for the global optimum design of composite modular structures - application to the design of an aircraft wing. Part 2: numerical aspects and examples. J Optim Theory Appl 2012;155(1):24-53.

[22] Tsai SW, Hahn T, Introduction to composite materials, Technomic; 1980

[23] Gibson LJ, Ashby MF. Cellular solids - structure and properties. Cambridge University Press; 1997.

[24] Montemurro M, Vincenti A, Vannucci P. Design of elastic properties of laminates with minimum number of plies. Mech Compos Mater 2012;48: 369-90.

[25] Jones RM. Mechanics of composite materials. McGraw-Hill; 1975

[26] Vannucci P, Verchery G. Stiffness design of laminates using the polar method. Int J Solids Struct 2001;38(50):9281-94.

[27] Catapano A, Stiffness and strength optimisation of the anisotropy distribution for laminated structures [Ph.D. thesis]. UPMC, Paris VI, France. <http:// tel.archives-ouvertes.fr/tel-00952372>; 2013.

[28] Vannucci P. A note on the elastic and geometric bounds for composite laminates. J Elast 2013;112:199-215.

[29] Montemurro M, Vincenti A, Vannucci P. The automatic dynamic penalisation method (ADP) for handling constraints with genetic algorithms. Comput Methods Appl Mech Eng 2013;256:70-87. 
[30] Montemurro M, Vincenti A, Koutsawa Y, Vannucci P. A two-level procedure for the global optimization of the damping behavior of composite laminated plates with elastomer patches. J Vib Control 2013:1-23. http://dx.doi.org/ $10.1177 / 1077546313503358$

[31] Montemurro M, Koutsawa Y, Belouettar S, Vincenti A, Vannucci P. Design of damping properties of hybrid laminates through a global optimisation strategy. Compos Struct 2012;94:3309-20.
[32] Montemurro M, Nasser H, Koutsawa Y, Belouettar S, Vincenti A, Vannucci P. Identification of electromechanical properties of piezoelectric structures through evolutionary optimisation techniques. Int J Solids Struct 2012 49(13):1884-92.

[35] HexWeb honeycomb attributes and properties, Duxford, Cambridge, UK; 1999. 